\title{
A database of computational models of a half-center oscillator for analyzing how neuronal parameters influence network activity
}

\author{
Anca Doloc-Mihu • Ronald L. Calabrese
}

Received: 30 September 2010 / Accepted: 6 January 2011 /

Published online: 12 February 2011

(C) Springer Science+Business Media B.V. 2011

\begin{abstract}
A half-center oscillator (HCO) is a common circuit building block of central pattern generator networks that produce rhythmic motor patterns in animals. Here we constructed an efficient relational database table with the resulting characteristics of the Hill et al.'s (J Comput Neurosci 10:281-302, 2001) HCO simple conductance-based model. The model consists of two reciprocally inhibitory neurons and replicates the electrical activity of the oscillator interneurons of the leech heartbeat central pattern generator under a variety of experimental conditions. Our long-range goal is to understand how this basic circuit building block produces functional activity under a variety of parameter regimes and how different parameter regimes influence stability and modulatability. By using the latest developments in computer technology, we simulated and stored large amounts of data (on the order of terabytes). We systematically explored the parameter space of the $\mathrm{HCO}$ and corresponding isolated neuron models using a brute-force approach. We varied a set of selected parameters (maximal conductance of intrinsic and synaptic currents) in all combinations, resulting in about 10 million simulations. We classified these HCO and isolated neuron model simulations by their activity characteristics into identifiable groups and quantified their prevalence. By querying the database, we compared the activity characteristics of the identified groups of our simulated HCO models with those of our simulated isolated neuron models and found that regularly bursting neurons compose only a small minority of functional HCO models; the vast majority was composed of spiking neurons.
\end{abstract}

Keywords Bursting - Oscillation - Central pattern generator - Database •

Parameter variation - Simulation - Isolated neuron - Automated analysis $\cdot$ Large datasets

\footnotetext{
A. Doloc-Mihu $(\varangle) \cdot$ R. L. Calabrese

Department of Biology, Emory University, Atlanta, GA 30322, USA

e-mail: adolocm@emory.edu
}

R. L. Calabrese

e-mail: biolrc@emory.edu 


\section{Introduction}

One approach to understanding how the brain works is to select a behavior and ask how the properties of neurons and their interconnections produce that behavior [2]. This approach has been especially successful in studying invertebrate animals with relatively simple nervous systems, such as the leech. Adaptive rhythmic behaviors such as walking and breathing are produced by central pattern-generating networks (CPG); CPGs are particularly well understood in invertebrates. The combination of the intrinsic electrical properties of the component neurons and their synaptic interactions within a CPG produces rhythmic activity [3]. The adaptability of these behaviors results from neuromodulatory and sensory inputs to CPGs and motor neurons.

Bursting activity, frequently observed in CPGs [3, 4], is characterized by intervals of repetitive spiking separated by intervals of quiescence. Single neuron oscillators and oscillatory circuit building blocks are important for burst generation by CPGs $[3,5]$. Studies of half-center oscillators (HCOs), which are constructed with reciprocally inhibitory neurons and are one of the most prevalent circuit building blocks of CPGs, show that they can display a wide range of bursting activity when the parameters controlling intrinsic membrane properties and synaptic interactions of the neurons are varied [3, 6-9]. The analysis of this basic circuit building block has helped us to understand how bursting activity is generated and how motor patterns are controlled by the nervous system. Moreover, it has elucidated general mechanisms of neural network function.

Computational modeling has been essential to the analysis of HCOs and busting activity $[6,10,11]$ by generating predictions that guide further experimentation to validate and refine the models and to derive new insights [12]. Computational manipulation of system parameters allows exploration of large spaces of parameter values that otherwise are not experimentally accessible. Such exhaustive explorations of parameter space help researchers understand the role of parameters (e.g., synaptic interactions) in bursting and CPG function. For example, by automatically exploring a parameter space of 20 million parameter combinations in a model of a simple CPG network, Prinz et al. [7] found that many different combinations of synaptic strengths and intrinsic membrane properties can produce similar network behavior.

During the process of parameter space exploration, very large amounts of data are generated. These large datasets can be automatically analyzed by taking advantage of relational databases. Although databases have been successfully used for decades in other fields $[13,14]$, in neuroscience their use for storing, managing, and analyzing data has been more recent and less sophisticated [15-17]. The results of the first fully automatic analysis of 20 million neuronal network models (done by Prinz et al. [15]) were recorded into a database consisting of text files, which are inefficient for storage, parsing, and querying.

Our study here is preliminary to a full investigation of how intrinsic membrane and synaptic parameters affect the electrical activity of a HCO model and how different parameter regimes influence stability and modulatability of the HCO model's output. For our study, we used the HCO computational model of Hill et al. [1], which was successfully developed to replicate the electrical activity of the oscillator interneurons of the leech heartbeat CPG under a variety of experimental conditions [1, 18, 19]. The complete, canonical HCO neuron model is a challenge for a comprehensive analysis of parameter variation. However, by using the latest developments in computer technology, we were able to simulate and store large amounts of data (on the order of terabytes). To explore systematically the parameter space of the $\mathrm{HCO}$ and corresponding isolated neuron models, 
we used the brute-force approach $[12,15,20,21]$. We varied a set of selected parameters (maximal conductance of intrinsic and synaptic currents) in all combinations resulting in about 10 million simulations. We then classified these HCO and isolated neuron model simulations by their activity characteristics, so that models showing the same electrical activity are segregated to the same group. We built a relational database table with the resulting model characteristics and determined the number of simulations in each activity group. Our database representation is much more efficient than databases that store and query raw data from text files. By efficiently querying the database, we also compared the activity characteristics of the groups of our HCO simulated models with the groups of our simulated isolated neuron models.

\subsection{Heartbeat central pattern generator of the leech}

In leeches, blood is moved through the closed circulatory system [22] by two lateral heart tubes. Segmental heart motor neurons that control heart contractions [23] are in turn controlled by rhythmic inhibitory input from a network of heart interneurons $(\mathrm{HN})$ that compose the heartbeat central pattern generator (CPG). Rhythmic activity in the heartbeat CPG is based on alternating bursting in two pairs of inhibitory interneurons that make reciprocal spike-mediated and graded synapses across the ganglionic midline [1] (Fig. 1, left). Each of these pairs, which are located in midbody segmental ganglia 3 and 4, comprises a HCO. Since the two pairs show similar activity, we limited our study to the pair of $\mathrm{HN}(4)$ interneurons, called $\mathrm{HN}(\mathrm{L}, 4)$ and $\mathrm{HN}(\mathrm{R}, 4)$ (for the left and right cell, respectively).

\subsection{Half-center oscillator model (Hill's model [1])}

For this study, we used the model of Hill et al. [1] of a HCO, which produces electrical activity similar to that observed in the living system. The HCO model consists of a pair of reciprocally inhibitory model neurons as shown in Fig. 1, left.

We represented the individual interneurons as single isopotential electrical compartments with Hodgkin and Huxley [24] type intrinsic and synaptic membrane conductances. Each compartment contains eight voltage-dependent currents, five inward currents $\left(I_{\mathrm{Na}}-\mathrm{a}\right.$ fast $\mathrm{Na}^{+}$current, $I_{\mathrm{P}}-$ a persistent $\mathrm{Na}^{+}$current, $I_{\mathrm{CaF}}-$ a rapidly inactivating low-threshold $\mathrm{Ca}$ current, $I_{\mathrm{CaS}}$ - a slowly inactivating low-threshold Ca current, and $I_{\mathrm{h}}$ - a hyperpolarizationactivated cation current), and three outward currents ( $I_{\mathrm{K} 1}-\mathrm{a}$ delayed rectifier-like $\mathrm{K}$ current, $I_{\mathrm{K} 2}-\mathrm{a}$ persistent $\mathrm{K}$ current, and $I_{\mathrm{KA}}-\mathrm{a}$ fast transient $\mathrm{K}$ current). The model has two types of inhibitory synaptic transmission between the two interneurons: graded transmission $\left(I_{\mathrm{SynG}}\right)$ and spike-mediated transmission $\left(I_{\mathrm{SynS}}\right)$. The graded transmission $I_{\mathrm{SynG}}$ was modeled as a postsynaptic conductance controlled by presynaptic $\mathrm{Ca}^{2+}$ concentration, and the spike-mediated transmission $I_{\text {SynS }}$ was modeled as a postsynaptic conductance triggered by presynaptic spikes. The differential equations of the model are given in the Appendix of [1]. Table 1 shows the values for the maximal conductances and the leak reversal potential that we used for our canonical model in this study.

Our immediate aim in this study was to explore how intrinsic membrane and synaptic parameters affect the electrical activity of the HCO model. In particular, we were interested in defining the parameter values that can lead to functional output from this circuit that conforms to that observed in the living system. To accomplish this aim, we created a model database of some 10,000,000 HCO model simulations in which key parameters indicated by 


\section{Example of a Simulated HCO Model}

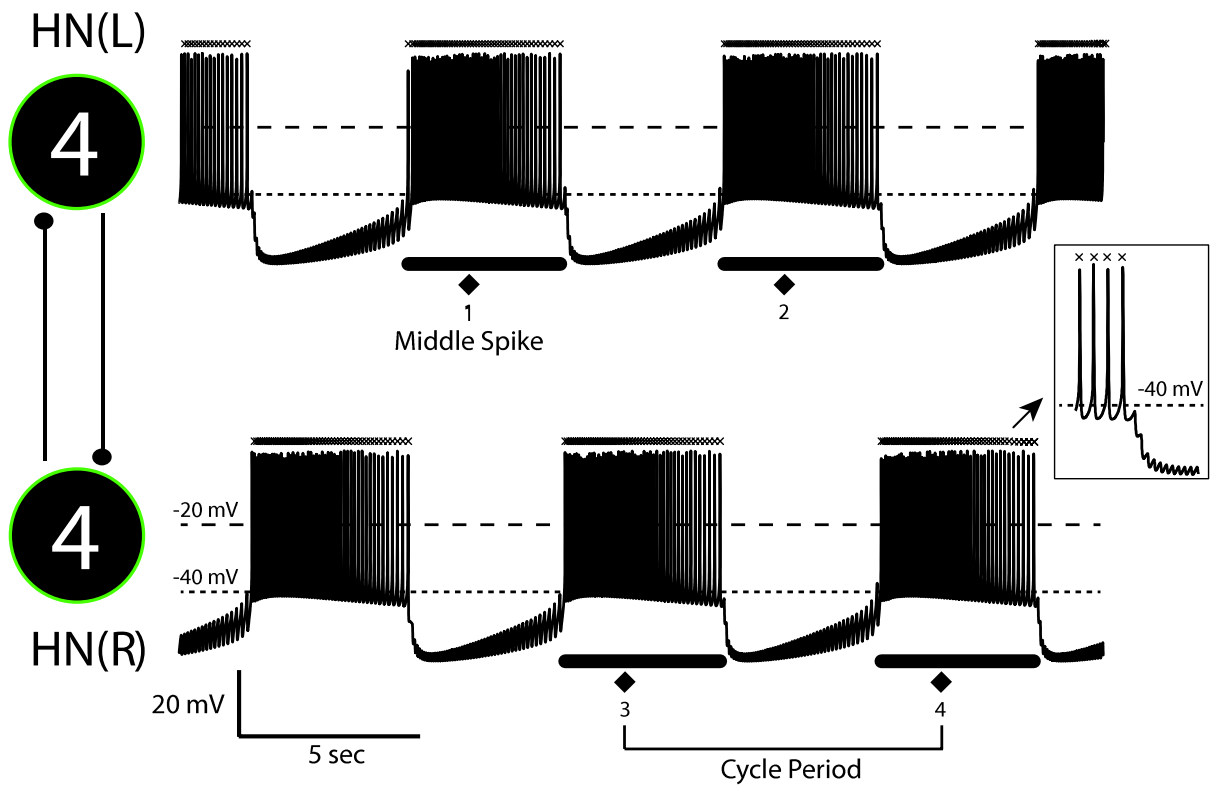

Fig. 1 HCO model of [1] (left) with two reciprocally inhibitory interneurons; the synaptic interactions are represented by small filled circles. Example of voltage traces from a simulated HCO model (right). Using our classification algorithm, each spike detected is labeled with a small cross, and the two bursts detected for each model neuron are labeled with bars and numbered ( 1 and 2 for the HN(L,4) model neuron and 3 and 4 for the $\mathrm{HN}(\mathrm{L}, 4)$ model neuron). The filled diamonds show the middle spikes of these numbered bursts; these middle spikes are used to compute period and phase in this system

gray shading in Table 1 were varied. We then classified these simulations by their activity characteristics. We also determined the activity characteristics of the component neurons of all HCOs.

\section{Methods}

\subsection{Definitions}

We defined a burst as having at least three spikes and a minimum inter-burst interval of $1 \mathrm{~s}$ (see Fig. 1, right). We defined the cycle period as being the interval between the middle spikes of two consecutive bursts $\left(\Delta_{L}=t_{2}-t_{1}\right.$, where $t$ is the time of the burst shown in

Table 1 Parameters of the canonical model: those shaded in gray were varied in constructing our model database. Parameters in the model other than maximal conductances and the leak reversal potential are considered fixed; see [1]

\begin{tabular}{llll}
\hline $\bar{g}_{\text {SynS }}=60 \mathrm{nS}$, & $\bar{g}_{\mathrm{K} 2}=80 \mathrm{nS}$, & $\bar{g}_{\mathrm{Na}}=200 \mathrm{nS}$, & $\bar{g}_{\mathrm{KA}}=80 \mathrm{nS}$, \\
$\bar{g}_{\mathrm{SynG}}=30 \mathrm{nS}$, & $\bar{g}_{\mathrm{CaS}}=3.2 \mathrm{nS}$, & $\bar{g}_{\mathrm{CaF}}=5 \mathrm{nS}$, & $\bar{g}_{\text {Leak }}=8 \mathrm{nS}$, \\
$\bar{g}_{\mathrm{P}}=7 \mathrm{nS}$, & $\bar{g}_{\mathrm{h}}=4 \mathrm{nS}$, & $\bar{g}_{\mathrm{K} 1}=100 \mathrm{nS}$, & $E_{\text {Leak }}=-60 \mathrm{mV}$ \\
\hline
\end{tabular}


Fig. 1, right for the left neuron). Phase was calculated on a per cycle basis, as being the delay from the middle spike of a burst of neuron $B$ to the middle spike of the preceding burst of neuron A divided by the interval from this middle spike of the next burst of neuron A to the middle spike of the preceding burst of neuron $\mathrm{A}\left(\phi=\frac{t_{4}-t_{1}}{t_{2}-t_{1}}\right)$. The duty cycle was defined as the percentage of the period occupied by a burst.

In the following, we define the labels for the HCO model groups (Section 2.3). These labels appear in the text in italics and correspond to the labels shown in Fig. 2 for the terminal groups of the tree structure. If the potential waveforms of a neuron's voltage trace did not cross a threshold of $-20 \mathrm{mV}$, then the neuron is considered to have silent activity. A HCO model has silent activity, if both its neurons have silent activities. If a neuron is not silent but does not have any recognizable bursts, then the neuron has spiking activity. A HCO model has a spiking activity, if both its neurons show a spiking activity. A neuron has a bursting activity if it has at least one burst in $100 \mathrm{~s}$. A HCO model has a bursting activity, if both its neurons have bursting activities. A HCO model has asymmetric activity, if its neurons have different types of activities (any combination of silent, spiking, and bursting).

If the voltage trace value at the end point of a burst is greater than $-35 \mathrm{mV}$, then the burst is considered a plateau. If a neuron has a plateau burst, then the neuron is considered as having plateau activity. A HCO model has a plateau activity, if both its neurons have plateau activities. If the coefficient of variation (defined as the ratio of the standard deviation to the mean) of the amplitudes of the spikes within a burst is greater than 0.07 , then the burst has irregular spikes; otherwise, the burst has normal spikes. A HCO model has irregular spikes activity, if both its neurons have irregular spikes activity. If all the bursts of a neuron have normal spikes, then the neuron is considered as having normal spikes type of activity. A HCO model has a normal spikes activity, if both its neurons have normal spikes activities. A HCO model has an asymmetric bursting activity, if its neurons have different types of bursting activities (any combination of plateau, irregular spikes, and normal spikes).

There are normal spikes neurons that have only one burst in a hundred seconds; if a $\mathrm{HCO}$ model has at least one such neuron, then the HCO model is defined as a one burst HCO model. Since we were interested in models that have at least two bursts (for which we could calculate period and phase), from now on, unless specifically noted, we assume that bursting $\mathrm{HCO}$ models have normal spikes neurons with more than two bursts.

If the coefficient of variation of the period (of a neuron) is less than 0.05 , then the neuron has regular period; otherwise, it has irregular period. A HCO model has an irregular period activity if at least one neuron has irregular period. A HCO model with both neurons having regular periods and phases in the range [0.45-0.55] is called a functional $\mathrm{HCO}$ model. If one of its phases is outside the above interval, then the model is called unbalanced.

We define a functional $\mathrm{HCO}$ as having two interneurons each showing bursting activity with normal spikes and repeated bursts, a small variation of period (coefficient of variation $\mathrm{cv}_{\text {Period }}<0.05$, regular period), relative phase in the range $0.45-0.55$ (balanced), and at least one synaptic component present.

Isolated neuron models Within the parameter variations of our database of simulations, we generated a group of models where both synaptic components have the parameter value 0 , i.e., $\bar{g}_{\text {SynS }}=0 \mathrm{nS}$ and $\bar{g}_{\text {SynG }}=0 \mathrm{nS}$. These correspond to models of synaptically isolated neurons, two identical ones per simulation, which we name isolated neuron models. Each isolated neuron model in these pairs is started with different initial conditions (see below), 


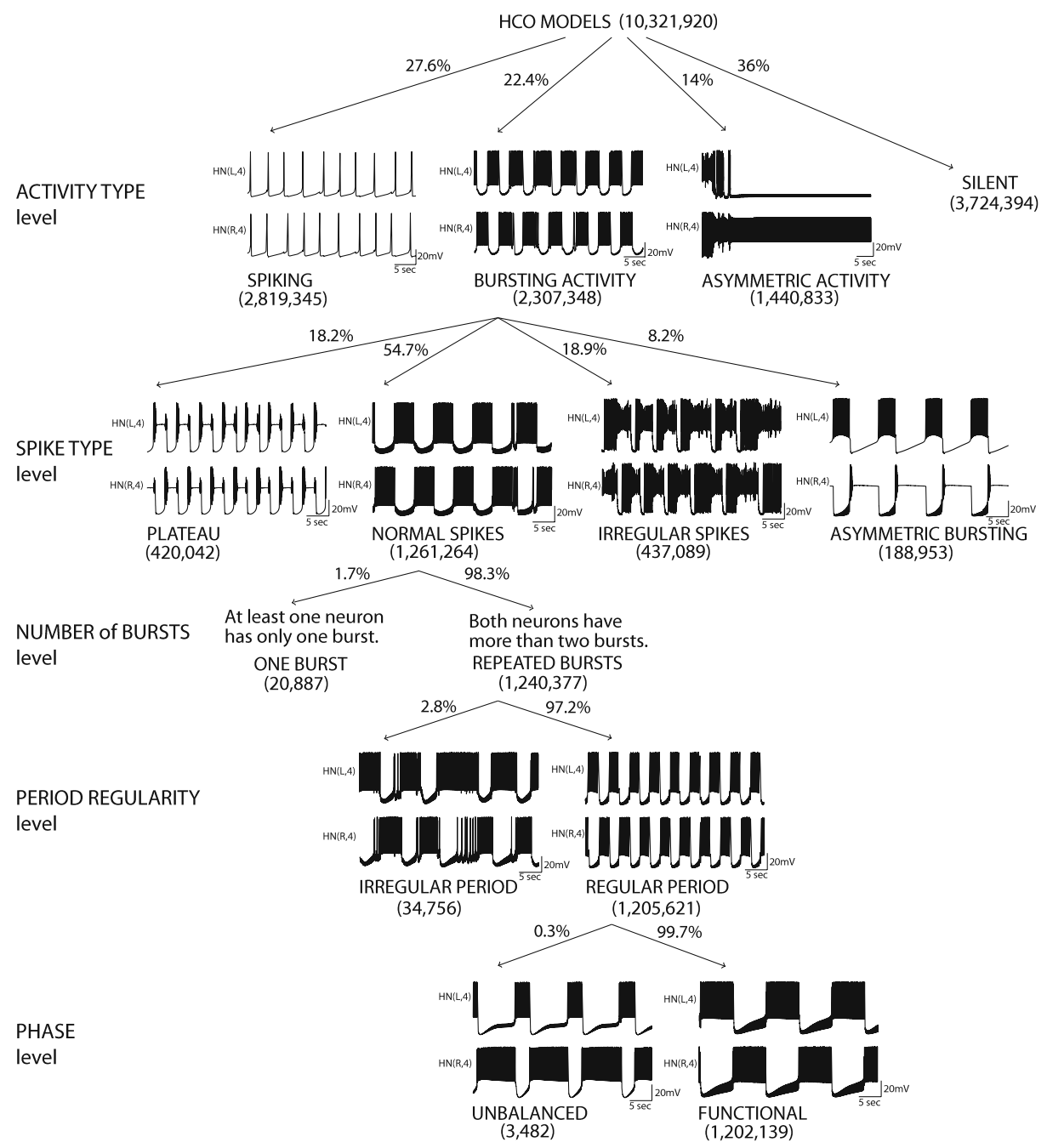

Fig. 2 Application of our classification method to HCO models in our database: A tree-like structure is obtained, in which the leaf nodes are ten main terminal groups of interest. At each step, represented as a level, the method splits a group into subgroups based on a specific criterion, which is indicated by the level's label. For each group, we illustrate an example of a simulated model (voltage traces of its neurons), the group label, the number of HCO models classified within the group, and its percentage of its parent group. An arrow indicates a group-subgroup relationship between two groups

but they are otherwise identical. For the isolated neuron models, we keep the definitions given above, with two exceptions: First, an isolated neuron model for which the two neurons have different types of electrical activities was named a bistable isolated neuron model. Then, an isolated neuron model for which both neurons have bursting, normal spikes activity, repeated bursts, and regular periods was named a regular bursting isolated neuron model. Note that for the isolated neuron models, there is no phase level or asymmetric bursting at the spike type level. 
Multistability In this study, we did not explore multistability directly, though we in fact know that multistability exists. For example, the HCO model with canonical parameters shows at least two activity regimes depending on initial conditions (irregular alternating spiking and regular alternating bursting) and can be easily switched between regimes by simulated current injection [1]. Because each simulation in this study was started from the same two sets of initial conditions (one for each model neuron) and no others were tried, we cannot know whether or not most of our models exhibit multistability. Nevertheless, multistability of various types appears likely in some cases, such as HCO models with asymmetric bursting activity. In isolated neuron models when the two model neurons in a pair show different activity, we may be assured that the model neuron exhibits at least bistability and hence our category name bistable isolated neuron model.

\subsection{Computational method}

For our study, we used the HCO model of Hill et al. [1] as described in Section 1.2. The differential equations of the model were integrated by using the exponential Euler method with a time step of $0.1 \mathrm{~ms}$ (or $10^{-4} \mathrm{~s}$ ) [1]. We ran our simulations in GENESIS version 2.3 [25]. The paired neurons $(\mathrm{HN}(\mathrm{L})$ and $\mathrm{HN}(\mathrm{R}))$ across all models used the same set of parameters. All simulations were started from the same initial conditions, which were different for each of the two neurons (Table 2). These initial conditions were obtained by running the canonical HCO model [1] for $200 \mathrm{~s}$, such that one of the two neurons was in its bursting state and the other one was being inhibited.

To investigate the changes in the HCO model's electrical activity and burst characteristics based on cellular and synaptic parameters, we varied eight selected parameters in all combinations by using a brute-force approach [26]. We used the same parameter values in each of the paired model neurons. We varied the maximal conductances of

Table 2 Initial conditions for the simulated models (see [1])

\begin{tabular}{lll}
\hline $\begin{array}{l}\text { Initial conditions for } \\
\text { each state variable in }\end{array}$ & $\mathrm{HN}(\mathrm{L})$ & $\mathrm{HN}(\mathrm{R})$ \\
the model equations & & \\
\hline$V_{\mathrm{m}}$ & -0.03989018337 & -0.04780111815 \\
$m_{\mathrm{Na}}$ & 0.162897651 & 0.05625188792 \\
$h_{\mathrm{Na}}$ & 0.9890171692 & 0.9999047991 \\
$m_{\mathrm{K} 1}$ & 0.06335179509 & 0.01806901543 \\
$h_{\mathrm{K} 1}$ & 0.7002682541 & 0.9241423938 \\
$m_{\mathrm{K} 2}$ & 0.242996044 & 0.07936307986 \\
$m_{\mathrm{KA}}$ & 0.6110944835 & 0.3354440828 \\
$h_{\mathrm{KA}}$ & 0.02548906894 & 0.1098876465 \\
$m_{\mathrm{h}}$ & 0.1099298106 & 0.4709321159 \\
$m_{\mathrm{CaF}}$ & 0.9685741383 & 0.1671418019 \\
$h_{\mathrm{CaF}}$ & 0.004908529471 & 0.1244102051 \\
$m_{\mathrm{CaS}}$ & 0.9477185297 & 0.2237872238 \\
$h_{\mathrm{CaS}}$ & 0.1060558064 & 0.3095398735 \\
$m_{\mathrm{P}}$ & 0.4511120959 & 0.2337070165 \\
$A(\mathrm{SynG})$ & $4.858213223 \mathrm{e}-12$ & $1.995938883 \mathrm{e}-11$ \\
$P(\mathrm{SynG})$ & $7.865283768 \mathrm{e}-13$ & $3.651243432 \mathrm{e}-12$ \\
$M(\mathrm{SynS})$ & 0.1003426686 & 0.5945098996 \\
$t_{\mathrm{S}}$ & -0.06390200555 & -3.691892624 \\
\hline
\end{tabular}


spike-mediated $\left(\bar{g}_{\text {SynS }}\right)$ and of graded transmission $\left(\bar{g}_{\text {SynG }}\right)$ and of the $I_{\mathrm{P}}, I_{\text {CaS }}, I_{\text {leak }}, I_{\mathrm{h}}$, and $I_{\mathrm{K} 2}$ currents, across of $0 \%, 25 \%, 50 \%, 100 \%, 125 \%, 150 \%$, and $175 \%$ of their canonical values (Table 1) and the leak reversal potential across $-70,-65,-60,-55$, and $-50 \mathrm{mV}$ values. Spike currents were not varied for two reasons: to limit the size of the parameter space and because they are not directly involved in the burst behavior. We focused only on maximal conductances because these parameters can be experimentally determined in our system and they had previously been shown to have strong and predictable effects on bursting behavior (as demonstrated by systematic variation of individual parameters in Hill et al. [1]). Kinetic parameters, such as half activation voltages, etc. are less easy to define experimentally and have not been systematically explored. We reserve their exploration for future study. Our parameter variation, albeit limited, resulted in a parameter space of 10,485,760 simulated models: 10,321,920 of these are HCO models because they have at least one synaptic component present, and the rest of them, 163,840, are essentially isolated neuron models but contain twin neurons without any synaptic interaction. We focused our research on the former set of 10,321,920 HCO models that had synaptic interaction (spikemediated and/or graded).

\subsubsection{Simulations}

After changing a parameter, we ran each model for $100 \mathrm{~s}$ to allow the system to establish stable activity, and then we ran it for another $100 \mathrm{~s}$, from which we recorded and analyzed the data. For each simulated model, we recorded the voltage traces of the electrical activity corresponding to its paired neurons for the first $40 \mathrm{~s}$ (GENESIS simulation time) of the simulation time into a voltage (binary) file. For each neuron of the simulated model, we recorded its spike times for the entire $100 \mathrm{~s}$ in an ASCII file. However, these simulations took different (total) computer running times depending upon the model's type of activity. Silent activity models took the least time (12 s), while functional HCOs with many bursts in both neurons took the most time (35 s); on average, each model took approximately $18 \mathrm{~s}$. The total simulation time was $52,429 \mathrm{~h}$. We ran our simulations on a Linux cluster with 125 nodes (each node having four CPUs, i.e., a total of $500 \mathrm{CPUs}$ ); the total running time was $105 \mathrm{~h}$ or approximately 4.5 days.

Although the operating system (Linux Fedora 9) on our computer permits 32,000 files per folder, we found that at approximately 10,000 files per folder the system's access to files became very slow. So, for fast access to our files, we decided to create an archive with our simulation files such that each folder contained a maximum of 6,300 files. That is, we stored our simulations files into folders and subfolders, with each subfolder having all the files corresponding to 1,050 simulated models.

Each simulated model had between four and six output files, depending on whether the model had silent activity or had one or both neurons with spiking activity. On average, the files of a simulated model occupied about $6 \mathrm{MB}$ space on disk. To save storage space, for each simulated model, we recorded its voltage traces and the conductances of $I_{\text {Syns }}, I_{\mathrm{h}}$, and $I_{\text {CaS }}$ of both neurons in a binary file (a binary file has a smaller size than an ASCII file); further, we used a disk_out format to be able to compress it. For compressing the binary (voltage) files, we used Free Lossless Audio Codec [27]. Note that the compression worked better for the silent, spiking, and asymmetric activity models (with compression rate of up to ten times) than for the bursting activity models (with compression rate of up to five to six times). The total size of the entire archive of the files of our 10,485,760 simulated models (including only the compressed voltage files) was $2.4 \mathrm{~TB}$. 


\subsubsection{Extracting firing characteristics}

Next, we analyzed these raw data by using our Matlab (http://www.mathworks.com/) scripts which implement the definitions given in Section 2.1. From a spike time file, we extracted the type of activity for that neuron: silent, spiking, or bursting activity. Note that we did not create a spike time file for a silent neuron.

From each neuron showing bursting activity (as defined in Section 2.1), we computed its firing characteristics: the number of bursts, the spike frequency (for which we calculated the mean and maximum values and their standard deviations), and the mean, standard derivation, and coefficient of variation of the period, duty cycle, and phase. For each simulated model, its firing characteristics were written into an ASCII file (size $1.3 \mathrm{kB}$ ). The scripts ran on six CPUs for approximately 16 days to parse the files of all the simulated models. Next, we built a Standard Query Language (SQL) database table (created with MySql, http://mysql.com/) with the resulting model characteristics similar to earlier work $[15,21]$.

\subsubsection{Database}

We used our own Java (Java 1.5) script to build a MySQL database and to record the characteristics and the parameters of each simulated model into this database. This construction simplifies our data analysis. The principal advantage of using the Java language is that it is platform-independent; thus, our scripts can run on different operating systems (Linux, Windows, Macintosh) without requiring any (re-coding) modifications. Another advantage of using Java is that when querying, a large table Java, unlike Matlab, does not require that the entire table be kept in memory; this feature is particularly useful when dealing with large databases (such as Pandora; see [17]). For each simulated model, the script read the characteristics of the model from its ASCII file (Section 2.2.2) and recorded them into a row of the database. Each model in the database has its own unique identifier (unique number which also is a primary key in the database), which makes it easier to query. In addition, for efficient querying, we used another 11 indexes: for each parameter, for each neuron type, and for the system type (group label).

We chose the optimal data type for each field in the database to minimize the storage space required and to speed up the querying process. For example, the parameter values were not recorded as floating point values but as small integers which gave the percents of the parameter values with respect to their canonical values. Also, we did not record the spike files and the voltage files into the database; we just recorded a pointer to the place in the archive where they reside (the name of the folder/subfolder for each model). Our Java script ran on one CPU for approximately $40 \mathrm{~h}$ to parse the ASCII files containing the characteristics of our simulated models and to record these characteristics into the MySQL database. Note that to speed up the database recording process, we inserted our data in batches of 1,000 records (each corresponding to a simulated model) into the database. We found that this process was time-consuming if every record was to be inserted either one at a time or simultaneously into the database. Also, we found that trying to insert batches of more than 1,500 records into the database was quite slow. So, we limited our batches to 1,000 records (models). The recorded data occupied 2.031 GB and the indexes occupied $1.103 \mathrm{~GB}$; our database has a total size of 3.134 GB. Our database is freely available via two DVDs mailed upon request; in the future, we will like to have it available on a web server. Our code is freely available on the ModelDB [28]. 
Then, for each simulation showing bursting activity in both neurons, we investigated its voltage file and grouped together those models showing similar bursting activity, with the goal of classifying all the simulated HCO models showing functional activity into the same group. For this task, we built our own classification method described in Section 2.3 below.

\subsection{Classification method}

Our classification method was designed such that there were no positive misses (for example, we did not classify models with bursting activity within the group of models with spiking activity), but we could have false negatives (for example, we may classify models with spiking activity within the group of models with bursting activity).

To develop our classification method, we sampled a random subset $(10,000)$ of our HCO models. We determined that the sample subset could be split into several groups such that simulated models with the same activity share the same group label. We assigned a unique label to each group. Once a simulated HCO model was classified, the method assigned it a label corresponding to the definitions (Section 2.1), which was also recorded into the SQL database. In addition, during a HCO model's classification process, its component neurons received their own labels, which were also recorded into the SQL database. The eight activity characteristics used as classification criteria by our algorithm are given in Appendix 3.

Step 1. Classifying models into silent, spiking, bursting activity, or asymmetric activity groups Our method parsed the spike time files of the neurons of the HCO models from the sample subset to assign a label to each neuron as follows: 'silent' to the neurons that did not have a spiking time file, 'bursting activity' to the neurons that showed at least one burst (see Section 2.1), and 'spiking' to the neurons that had spikes but did not have any recognizable burst according to our definition. Then, the method assigned a label to each HCO model as follows: silent, spiking, or bursting activity to these models that had both neurons labeled 'silent', 'spiking', or 'bursting activity', respectively, and asymmetric activity to those models with their two neurons labeled different from each other.

Step 2. Classifying bursting activity models into plateau, normal spikes, irregular spikes, or asymmetric bursting activity groups We analyzed the voltage trace of each burst for each neuron in a HCO model to group HCO models with bursting activity into: plateau, irregular spikes, and normal spikes (Section 2.1). For each burst of a neuron, the method checked first for the plateaus (the value of the voltage trace at the end point of the burst was greater than $-35 \mathrm{mV}$ ) and irregular spikes (coefficient of variation of the amplitudes of the spikes was less than or equal to 0.07 ) criteria. The algorithm stopped at the first burst of a neuron for which either criterion was satisfied and labeled the neuron as belonging to that group. If, for a neuron, the algorithm reached the end point of its last burst without assigning it either of these labels, then the algorithm labeled the neuron with the normal spikes label. That is, the neuron had only bursts with normal spikes, and it had neither plateaus nor bursts with irregular spikes. Then, if the two corresponding neurons of a model received the same label, the model was labeled with the same label; otherwise, the model was labeled as asymmetric bursting.

After applying these classifiers, we found that for the sample subset, both neurons produced plateaus in $16 \%$ of the models. Note that in our classification process a neuron 
with spikeless plateau activity would be classified as spiking activity neuron. In $20 \%$ of the models, both neurons had irregular spikes activity determined from the spikes' amplitudes. In $62 \%$ of the subset (or $10.85 \%$ of the entire set), both neurons had normal spikes activity, and the remaining of $2 \%$ had asymmetric bursting activity. We next investigated in more detail the set of the models with both neurons having bursting, normal spikes activity.

Step 3. Selecting the models for which both neurons have repeated bursts This method separated out those HCO models (one burst models) for which at least one neuron had only one burst from the other models in the bursting models with normal spikes group, thus identifying those models for which both neurons had repeated bursts. This separation was done easily and efficiently by querying the SQL database for the number of bursts in each neuron. Note that those models whose neurons had only one burst within the recorded voltage file of $40 \mathrm{~s}$ were re-simulated and a new voltage file was recorded for the entire duration of $100 \mathrm{~s}$. A re-simulated model was assigned to this one burst group only if even after $100 \mathrm{~s}$ at least one of its neuron(s) showed only one burst. At this step, the one burst models were separated from HCO models that were bursting, normal spikes, repeated bursts HCO models.

\section{Results}

\subsection{Sampling the database}

As a preliminary step in the analysis of the model database, we sampled the database by selecting a random sample of 10,000 models from the entire set, and for this subset, we built a SQL database table $[15,21]$ of their firing characteristics. In approximately $37 \%$ of these simulations, both neurons were silent. In approximately $30 \%$ of these simulations, both neurons were spiking. Approximately $15.5 \%$ of the simulations had asymmetric activity in the two neurons. In $17.5 \%$ of the simulations, both neurons had bursting activity (see Section 2.1).

\subsection{Groups of HCO simulated models}

Our first step in the analysis of the models in our database was to group together all the simulated HCO models showing the same electrical activity. For this task, we built an algorithm (see Appendix 1) capable of automatically assigning a group label to each simulated model, such that models with same electrical activity share the same group label. The algorithm parsed the spike and voltage files corresponding to each simulated HCO model and gathered information about its electrical activity. Based on this information, it assigned the group labels, which were recorded into the SQL database in a separate column named "systType" shown also in Fig. 2. In this way, we were able to access diverse statistics about these groups quickly by just querying the database. Initially, the grouping algorithm was built by inspecting a random sample of 10,000 simulated models, and then, it was refined and applied to the entire set of simulated HCO models.

By using this algorithm, we divided the simulated HCO models in the entire database into several main groups. The results are illustrated in Fig. 2. The figure shows an example 
of a simulated model from each group. For each model displayed, the electrical activities of both neurons are plotted along with the group label, the number of models within the respective group, and the percentage of the respective group within its immediate parent group. An arrow indicates the relationship between groups; if a group is split into several sub-groups, then an arrow is drawn from the parent group to each of its children's groups. In this way, the results of the classification method are displayed in a tree-like structure in which the leaf nodes (terminal groups) are the main groups of interest.

The first three levels in Fig. 2 show the groups obtained after applying each step of our method described in Section 2.3. At the first step, the database was split into four main groups: the group of spiking HCO models representing $27.6 \%$ of the entire database, the group of bursting HCO models representing $22.4 \%$ of the database, the group of silent $\mathrm{HCO}$ models representing $36 \%$, and the group of asymmetric activity HCO models representing $14 \%$ of the database. At the second step, the group of bursting HCO models was subdivided by our classification method into four main groups: $18.2 \%$ plateau $\mathrm{HCO}$ models, $18.9 \%$ irregular spikes HCO models, 8.2\% asymmetric bursting HCO models, and $54.7 \%$ normal spikes HCO models. At the next step, the bursting, normal spikes group of HCO models was divided into two groups: $1.7 \%$ one burst $\mathrm{HCO}$ models that have at least one neuron showing only one burst in $100 \mathrm{~s}$ of simulation time and $98.3 \%$ for which both neurons showed repeated bursts (two or more bursts of the normal spikes). This latter group of bursting, normal spikes, repeated bursts HCO models comprises 1,240,377 models or approximately $12.02 \%$ of the entire $\mathrm{HCO}$ model database and also contains the functional HCO models that we are most interested in. According to our definition (see Section 2.1), a functional model must satisfy two electrophysiological criteria observed in living HN(4) HCOs: a regularity criterion given by the variation in the period together with a balance criterion given by the phase. By querying our SQL database with these two criteria, we obtained efficiently the functional HCOs from the bursting, normal spikes, repeated bursts HCO models. First, the regularity criterion divided the group of bursting, normal spikes, repeated bursts $\mathrm{HCO}$ models into two groups: $2.8 \%$ with irregular period and $97.2 \%$ with regular period. Then, the balance criterion divided these regular period $\mathrm{HCO}$ models into unbalanced activity models $(0.3 \%)$ and functional activity models $(99.7 \%)$. The process and the associated terminal groups are illustrated in Fig. 2 at the last two levels of the tree. At this point, we have the group of functional HCO models along with nine other groups, showing the ten different main electrical activities existing in our database of simulated HCO models.

Figure 3 illustrates, for each terminal group (the leaves of the tree) of HCO models at each level of the classification tree (Fig. 2), the number of models in the group and their percentage within the entire database. At the activity type (first) level, in 3,724,394 HCO models ( $\sim 36 \%)$, both neurons were silent; in 2,849,345 ( 27.6\%), both neurons were spiking; and in 1,440,833 (14\%) of the HCO models, the two component neurons showed asymmetric activity. At the spike type (second) level in the tree of Fig. 2, in 420,042 HCO models $(\sim 4 \%)$, the component neurons produced plateaus; in 437,089 HCO models $(4 \%)$, both neurons showed irregular spikes activity; and in 188,953 (2\%) models, the neurons showed asymmetric bursting activity. At the number of bursts (third) level of the tree, 20,887 HCO models were one burst models (less than 1\%). At the period regularity (fourth) level of the tree, 34,756 HCO models had irregular periods (less than 1\%). At the phase (fifth) level of the tree, 3,482 were unbalanced HCO models (less than 1\%) and 1,202,139 HCO models $(\sim 12 \%)$ of the total showed functional HCO activity. 


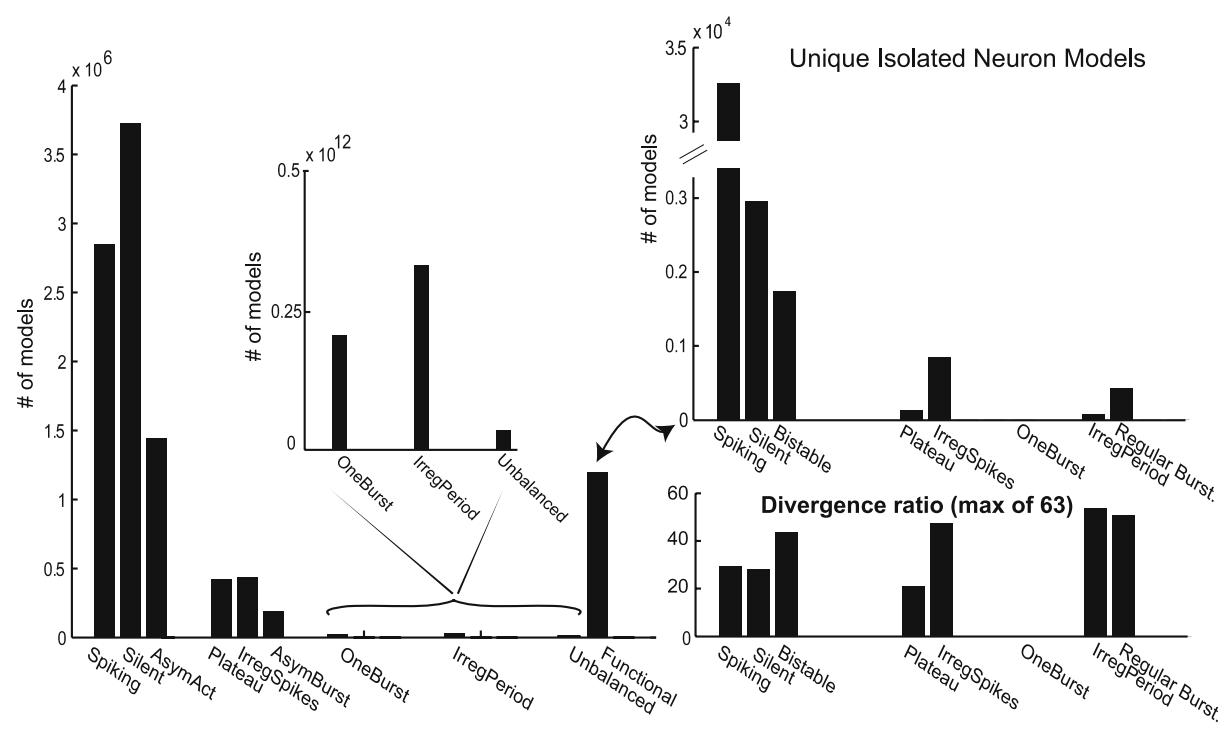

Fig. 3 Groups statistics within the database of 10,321,920 simulated HCO models

\subsection{Intrinsic properties of $\mathrm{HCO}$ model neurons}

We next wanted to analyze the intrinsic properties, independent of synaptic interaction, of the model neurons that make up all the HCO models simulated. In our total set of simulated models, there were 163,840 models of two neurons without either synaptic component (spike-mediated or graded) present, which we named isolated neuron models. In essence, each of these isolated neuron models contains two different instantiations of single neuron models with identical parameters but different initial conditions. These model neurons correspond to the model neurons of the HCO models as will be described below, but first we describe their intrinsic properties.

\subsubsection{Activity groups of isolated neuron models}

For the isolated neuron models, we designated the following eight groups (illustrated in Fig. 4): at the activity type (first) level of classification, spiking, silent, bistable, bursting groups; further parsing the bursting group at the spike type (second) level, we have plateau, irregular spikes, asymmetric, and normal spikes subgroups; further parsing the normal spikes subgroup at the number of bursts (third) level, we have one burst and repeated bursts subgroups; further parsing the repeated bursts group at the period regularity (fourth) level, we have irregular period and regular bursts (period) subgroups. Figure 4 illustrates the statistics for our simulated isolated neuron models. Most of them $(98,846)$ had silent neurons. Many of them $(56,904)$ showed both neurons with spiking activity. In 6,373 of them, the two neurons showed different electrical activity, i.e., they showed bistable activity. Of those with bursting activity (1,717), 265 showed plateau activity, 952 of them showed irregular spikes activity, and the rest showed normal spikes activity. Of those with bursting, normal spike activity, 76 showed an irregular period and 424 had both their neurons with 


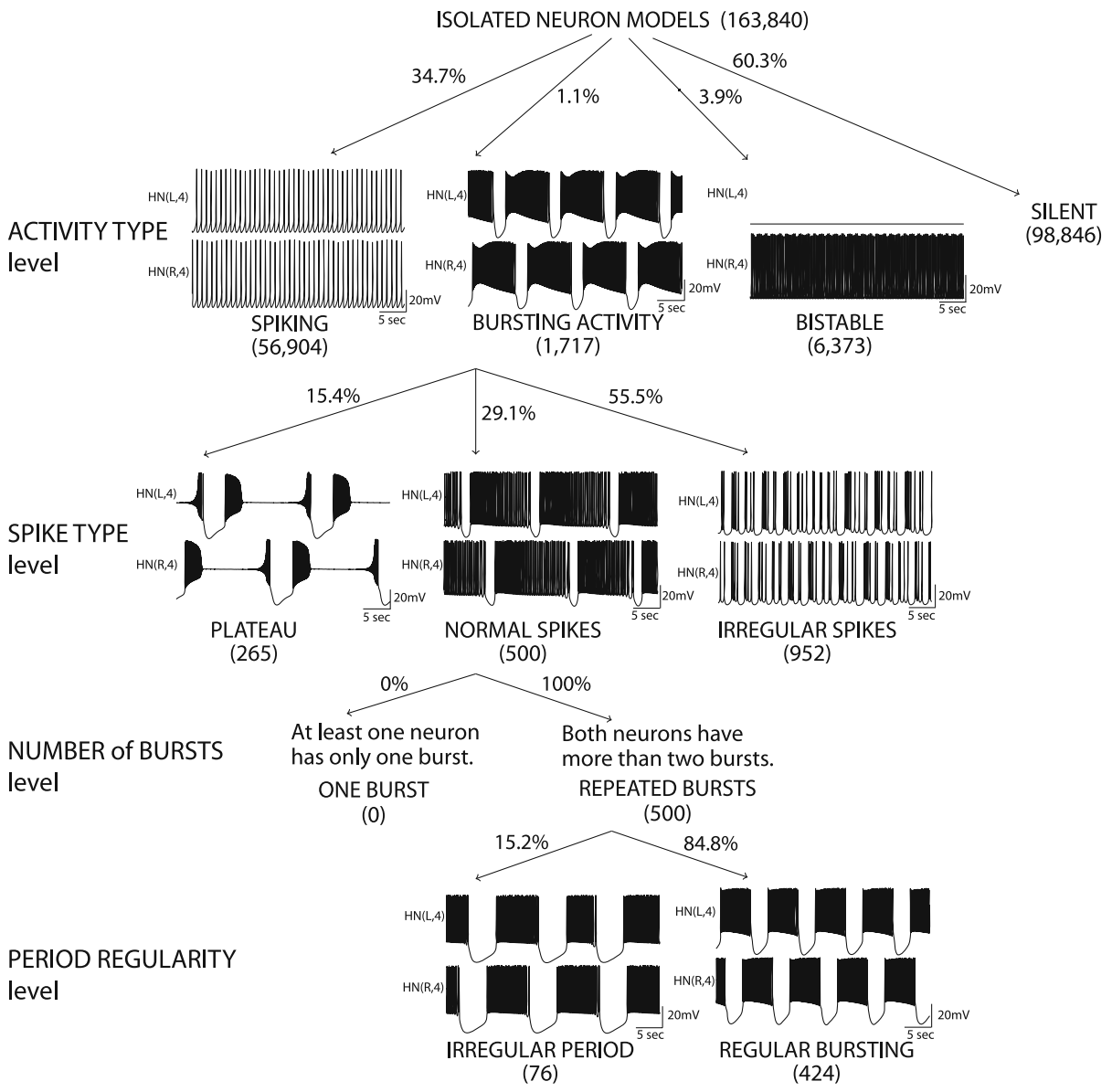

Fig. 4 Application of our classification method to isolated neuron models in our database: A tree-like structure similar to the one in Fig. 2 for HCOs is obtained, in which the leaf nodes are eight main terminal groups of interest. At each step, represented as a level, the method splits a group into subgroups based on a specific criterion, which is indicated by the level's label. For each group, we illustrate an example of a simulated model (voltage traces of its neurons), the group label, the number of isolated neuron models classified within the group, and its percentage of its parent group. An arrow indicates a group-subgroup relationship between two groups.

regular period, i.e., they were regular bursting isolated neuron models. The regular bursting isolated neuron models correspond to endogenous bursting neurons in biological systems.

\subsubsection{Correspondence between isolated neuron models and HCO models}

We simulated a total of 10,485,760 models, of which 10,321,920 were HCO models (at least one synaptic component present) and 163,840 were isolated neuron models (without any synaptic interaction). However, there is a correspondence of 1 to 63 between these two sets of models, i.e., for each isolated neuron model, there are $63 \mathrm{HCO}$ models that have 
exactly the same combination of non-synaptic parameter values as the one of the isolated neuron model.

To determine the intrinsic activity of the model neurons in our HCO models, we compared the activity of the $\mathrm{HCO}$ model neurons (that have at least one synaptic intrinsic component present (value 0)) and the corresponding isolated neuron model with the same non-synaptic parameters. However, since we wanted to have this type of results for the entire database of models, querying the database was a time-consuming task. Therefore, we built a Java script to query the entire database only once for this model correspondence task and built a new database in which each record is a pair of such corresponding models; this new database was queried for this task. The SQL query syntax for this correspondence task is given in Appendix 2. The new database sped up the querying process, since it contains only the needed information of the paired models. This database contains 10,321,920 records (i.e., each model with synaptic interaction(s) has one corresponding model without any synaptic interaction in the database). Table 4 (Appendix 5) shows the number of models within each activity group for both $\mathrm{HCO}$ and isolated neuron models.

For the group of functional HCO models, the statistics of their corresponding single cell models is illustrated in Fig. 3 (right). The group has 21,303 HCO models, which are made up from regular bursting neurons (419 unique models), i.e., from corresponding isolated models with the same non-synaptic parameters. The functional HCOs group also includes 81,701 HCO models made up from corresponding silent isolated neuron models (2,943 unique models), 978,151 HCO models made up from corresponding spiking isolated neuron models (32,568 unique models), 2,435 HCO models made up from corresponding plateau isolated neuron models (120 unique models), 40,703 HCO models made up from corresponding irregular spikes isolated neuron models (838 unique models), 3,951 HCO models made up from corresponding irregular period isolated neuron models (76 unique models; these isolated neuron models show bursting with irregular period), and 73,895 HCO models made up from corresponding bistable isolated neuron models (1,749 unique models).

If we look at the number of unique isolated neuron models, we notice that approximately seven times more silent isolated neuron models than regular bursting isolated neuron models (i.e., models that correspond to endogenously bursting neurons in biological systems) produce functional HCO models; 77.7 times more spiking isolated neuron models produce functional HCOs than regular bursting isolated neuron models; 4.17 times more bistable isolated neuron models produce functional HCOs than regular bursting isolated neuron models; two times more irregular spikes isolated neuron models produce functional HCOs than regular bursting isolated neuron models. On the other hand, 3.49 times fewer plateaus isolated neuron models produce functional HCOs than regular bursting isolated neuron models, and 5.5 times fewer irregular period isolated neuron models produce functional HCOs than regular bursting isolated neuron models. This analysis indicates that the vast majority of our functional HCO models are made up from spiking neuron models with a significant minority made up of silent or bistable isolated neuron models and only a small minority made up of regular bursting isolated neuron models.

Looked at in a different way, however, the contribution of regular bursting isolated neuron models to functional HCO models seems more important; a given regular bursting isolated neuron produces on average about 50.8 functional $\mathrm{HCO}$ models close to the limit of 63. In contrast, this divergence ratio is 30.0 for spiking, 27.8 for silent, 42.2 for bistable, 20.3 for plateau, 48.6 for irregular spikes, and 52.0 for irregular period isolated 
neuron models. The results also show that irregular period and irregular spiking neurons produce more functional HCOs than unbalanced HCOs. So, it appears that inhibition can clean up irregular activity into regular bursting; exploring the mechanisms by which this regularization is achieved will require much future work.

\section{Discussion and conclusions}

We are now in a position to begin to ask questions of our models that have important biological implications and that can be answered by simply querying our database. For example, our analysis of the correspondence between functional HCOs and the isolated neurons that make them up, though at a very early stage, still reveals some important insights. The data in Fig. 3 indicate that, within the parameter space we considered for the isolated neuron models, the spiking neurons are highly prevalent, suggesting that this mode of activity is robust. Regular bursting neurons are, on the other hand, rare, suggesting that this state requires a delicate balance of intrinsic membrane conductances (parameters) and that they may not be robust to parameter changes. This latter conclusion fits with the observations in the living system that pharmacologically isolated oscillator heart interneurons may be regular bursters, but they are very sensitive to small changes in leak conductance and leak reversal potential, which tend to move them into the spiking state [19]. Nevertheless, our same data show that regular bursting isolated neurons form functional HCOs that are apparently more robust to variations in synaptic parameters (divergence ratio $=50.8$ ) than those formed by spiking isolated neurons (divergence ratio $=$ 30.0). By this criterion, it is surprising to note the comparable robustness to synaptic parameter variation in HCOs formed from silent and from spiking isolated neurons. The robustness to variation in synaptic parameters is of particular interest to us because heart interneuron HCOs in the living system display a large animal-to-animal variation in synaptic conductance without apparent change in their activity [29]. In future studies, we will use the database developed here to explore in depth the interaction of parameters that lead to the different activity groups we have identified, how synaptic interactions transform the activity characteristics of isolated neurons, and how robustness and modulatability differ among $\mathrm{HCO}$ (or isolated neuron) models with similar activity but different parameters.

Acknowledgement This work was supported by the National Institute of Neurological Disorders and Stroke Grant NS024072 to R.L. Calabrese.

\section{Appendix 1: Group classification algorithm}

Pseudocode

For each simulated model do

(A) assign label to each of the two neurons corresponding to their activity type;

(B) assign label group based on both neurons' activity type;

end for; 


\section{Step 1-Activity type level}

- for both neurons of a model do

- $\quad$ if a neuron doesn't have a spiking file created, assign channelType = 'silent';

- else parse its spiking file

- $\quad$ if the neuron doesn't show any burst, assign channelType = 'spiking';

- $\quad$ else assign channelType = 'bursting activity';

end for;

- $\quad$ assign to the model its group label

- $\quad$ if its neuron labels are different, assign systType = 'asymmetric activity'; done.

- else

- assign systType $=\{$ 'spiking', 'silent' $\}$ if the labels of both neurons are channelType $=\{$ 'spiking', 'silent' $\}$, respectively; done.

- or assign systType $=\{$ 'bursting activity' $\}$ if its neuron labels are channelType $=$ 'bursting activity'; go to Step 2;

\section{Step 2-Spike type level}

- $\quad$ for each neuron of a model labeled 'bursting activity' do

- $\quad$ for each of its bursts, check

- $\quad$ if a burst is plateau, then re-assign channelType = 'plateau';

- $\quad$ else if a burst has irregular spikes, then re-assign channelType = 'irregular spikes';

end for (burst checking);

- if neuron's label is neither 'plateau' nor 'irregular spikes', then re-assign channelType = 'normal spikes';

end for;

- $\quad$ assign to the model its group label

- $\quad$ if its neurons' labels are different, re-assign systType = 'asymmetric bursting'; done.

- else

- re-assign systType $=\{$ 'plateau', 'irregular spikes' $\}$ if the labels of both neurons are channelType $=\{$ 'plateau', 'irregular spikes' $\}$, respectively; done.

- $\quad$ or re-assign systType $=\{$ 'normal spikes' $\}$ if its neuron labels are channelType $=$ 'normal spikes'; go to Step 3;

\section{Step 3-Number of bursts level}

- $\quad$ for each neuron of a model labeled 'normal spikes' check its number of bursts

- $\quad$ if number_of_bursts $=1$, re-assign channelType = 'one burst';

- $\quad$ else re-assign channelType = 'repeated bursts';

end for; 
- $\quad$ assign to the model its group label

- $\quad$ if its neurons' labels are 'repeated bursts', then re-assign systType $=$ ' repeated bursts'\}; go to Step 4;

- $\quad$ else re-assign systType $=\left\{{ }^{\prime}\right.$ one burst' $\}$; done

\section{Step 4-Period regularity level}

- $\quad$ for each neuron of a model labeled 'repeated bursts' check the coefficient of variation (cv) of its period

- $\quad$ if $\mathrm{cv}>=0.05$, re-assign channelType $=$ 'irregular period';

- $\quad$ else re-assign channelType = 'regular period';

end for;

- $\quad$ assign to the model its group label

- $\quad$ if both its neurons' labels are 'regular period', then re-assign systType $=$ ' 'regular period' \}; go to Step 5;

- $\quad$ else re-assign systType $=\{$ 'irregular period' $\}$; done.

\section{Step 5-Phase level}

- $\quad$ for each neuron of a model labeled 'regular period' check its phase range

- $\quad$ if phase in $[0.45,0.55]$, re-assign channelType = 'functional';

- $\quad$ else re-assign channelType = 'unbalanced';

end for;

- $\quad$ assign to the model its group label

- if both its neurons' labels are 'functional', then re-assign systType = \{'functional'\}; done.

- $\quad$ else re-assign systType $=\{$ 'unbalanced' $\}$; done.

\section{Appendix 2: SQL query}

\section{SELECT}

eo1. 'simNo' as simNo_1, eo1. 'simParPer_gBarSynS' as synS_1, eo1. 'simParPer_ gBarSynG' as synG_1, eo2. 'simNo' as simNo_2, eo2. 'simParPer_gBarSynS' as synS_2, eo2. 'simParPer_gBarSynG' as synG_2

FROM EO_db AS eo1 INNER JOIN EO_db AS eo2 USING ('simPar_EleakVal', 'simParPer_gBarP', 'simParPer_gBarK2', 'simParPer_gBarLeak', 'simParPer_gBarCaS', 'simParPer_gBarh')

WHERE ((eo1. 'simParPer_gBarSynG' $<>0$ or eo1. 'simParPer_gBarSynS' $<>0$ ) AND (eo2. 'simParPer_gBarSynG' $=0$ or eo2. 'simParPer_gBarSynS' $=0)$ ) 


\section{Appendix 3: Activity characteristics}

Our classification method split the HCO models into seven terminal groups at three different tree levels (Fig. 2): at level 1 spiking, silent, and asymmetric activity; at level 2 plateau, irregular spikes, and asymmetric bursting; at level 3 one burst. The repeated bursts HCOs group resulting from level 3 parsing was further split employing electrophysiological criteria into three terminal groups: at level 4 irregular period; at level 5 unbalanced and functional to obtain the functional HCOs group, which correspond to activity observed in the living system. In summary, during this classification process, there were eight activity characteristics used as follows:

1. Any spiking activity present or not (threshold of $-20 \mathrm{mV}$ ) to obtain the silent and non-silent HCOs

2. Number of spikes - presence of minimum three spikes to define a burst or not

3. Presence of a minimum inter-burst interval of $1 \mathrm{~s}$ to differentiate between spiking and bursting activities

4. The value of the voltage trace at the end point of the burst (greater than $-35 \mathrm{mV}$ ) to obtain the plateaus

5. Coefficient of variation of the amplitudes of the spikes in the burst $(\leq 0.07)$ to obtain the irregular spikes and normal spikes HCO models

6. The number of bursts per neuron in $100 \mathrm{~s}$ of simulation time to obtain the one burst models and the repeated bursts HCOs; the next two criteria were used to split the repeated bursts $\mathrm{HCOs}$

7. Coefficient of variation of the period $(<0.05)$ to obtain the irregular period and regular period models

8. Mean phase (in the range of $0.45-0.55$ ) to obtain the unbalanced and functional models

\section{Appendix 4: Synaptic components for each HCO group}

Table 3 Terminal groups for all HCO models broken down by the synaptic components present

\begin{tabular}{|c|c|c|c|c|}
\hline \multirow[t]{2}{*}{ Level } & \multirow{2}{*}{$\frac{\text { Group }}{\text { Terminal group }}$} & \multicolumn{3}{|l|}{ HCO models } \\
\hline & & Both synapses & SynS only & SynG only \\
\hline \multirow[t]{3}{*}{ Activity type } & Spiking & $2,125,786$ & 391,671 & 331,888 \\
\hline & Silent & $2,678,813$ & 386,642 & 658,939 \\
\hline & Asymm. activity & $1,226,954$ & 176,569 & 37,310 \\
\hline \multirow[t]{3}{*}{ Spike type } & Plateau & 409,666 & 6,822 & 3,554 \\
\hline & Irregular spikes & 351,650 & 43,096 & 42,343 \\
\hline & Asymm. bursting & 171,507 & 14,866 & 2,580 \\
\hline No. bursts & One burst & 18,193 & 2,684 & 10 \\
\hline Period regularity & Irregular period & 28,940 & 5,439 & 377 \\
\hline \multirow[t]{2}{*}{ Phase } & Unbalanced & 2,072 & 382 & 1,028 \\
\hline & Functional & $1,014,579$ & 118,709 & 68,851 \\
\hline Total & & $8,028,160$ & $1,146,880$ & $1,146,880$ \\
\hline
\end{tabular}




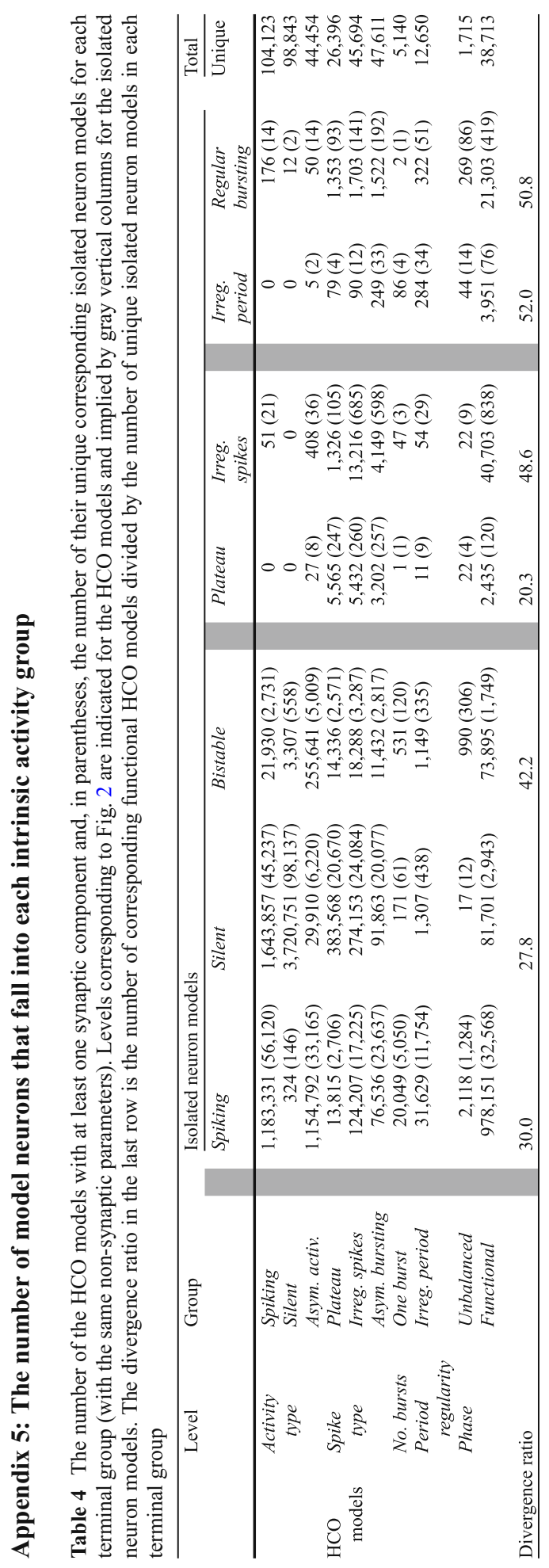




\section{References}

1. Hill, A.A.V., Lu, J., Masino, M.A., Olsen, O.H., Calabrese, R.L.: A model of a segmental oscillator in the leech heartbeat neuronal network. J. Comput. Neurosci. 10, 281-302 (2001)

2. Kristan, W.B. Jr., Calabrese, R.L., Friesen, W.O.: Neuronal control of leech behavior. Progr. Neurobiol. 76(5), 279-327 (2005)

3. Marder, E., Calabrese, R.L.: Principles of rhythmic motor pattern generation. Physiol. Rev. 76(3), 687717 (2004)

4. Cymbalyuk, G.S., Calabrese, R.L.: Oscillatory behaviors in pharmacologically isolated heart interneurons from the medicinal leech. J. Neurocomput. 32-33, 97-104 (2000)

5. Dean, J., Cruse, H.: Motor pattern generation. In: Arbib, M. (ed.) The Handbook of Brain Theory and Neural Networks, pp. 600-605. MIT, Cambridge (1995)

6. Marder, E., Bucher, D., Schulz, D.J., Taylor, A.L.: Invertebrate central pattern generation moves along. Curr. Biol. 15, R685-R699 (2005)

7. Prinz, A.A., Bucher, D., Marder, E.: Similar network activity from disparate circuit parameters. Nature Neurosci. 7, 1345-1352 (2004)

8. Olypher, A., Calabrese, R.L.: Using constraints on neuronal activity to reveal compensatory changes in neuronal parameters. J. Neurophysiol. 98, 3749-3758 (2007)

9. Olypher, A., Calabrese, R.L.: How does maintenance of network activity depend on endogenous dynamics of isolated neurons? Neural Comput. 21, 1665-1682 (2009). Erratum in: Neural Comput. 21, 2405 (2009)

10. De Schutter, E., Ekeberg, O., Kotaleski, J.H., Achard, P., Lansner, A.: Biophysically detailed modelling of microcircuits and beyond. Trends Neurosci. 28, 562-569 (2005)

11. Grillner, S., Kozlov, A., Dario, P., Stefanini, C., Menciassi, A., Lansner, A., Hellgren Kotaleski, J.: Modeling a vertebrate motor system: pattern generation, steering and control of body orientation. Prog. Brain Res. 165, 221-234 (2007)

12. Prinz, A.A.: Insights from models of rhythmic motor systems. Curr. Opin. Neurobiol. 16(6), 615-620 (2006)

13. Elmasri, R., Navathe, S.B.: Fundamentals of Database Systems, 2nd edn. Addison-Wesley, Menlo Park (1994)

14. Codd, E.F.: A relational model of data for large shared data banks. Commun. ACM 13(6), 377-387 (1970)

15. Prinz, A.A., Billimoria, C.P., Marder, E.: Alternative to hand-tuning conductance-based models: construction and analysis of databases of model neurons. J. Neurophysiol. 90, 3998-4015 (2003)

16. Calin-Jageman, R.J., Tunstall, M.J., Mensh, B.D., Katz, P.S., Frost, W.N.: Parameter space analysis suggests multi-site plasticity contributes to motor pattern initiation in tritonia. J. Neurophysiol. 98(4), 2382-2398 (2007)

17. Gunay, C., Edgerton, J.R., Li, S., Sangrey, T., Prinz, A.A., Jaeger, D.: Database analysis of simulated and recorded electrophysiological datasets with PANDORA's toolbox. Neuroinformatics 2(7), 93-111 (2009). http://userwww.service.emory.edu/ cgunay/pandora/

18. Cymbalyuk, G.S., Calabrese, R.L.: A model of slow plateau-like oscillations based upon the fast $\mathrm{Na}^{+}$ current in a window mode. J. Neurocomput. 38, 159-166 (2001)

19. Cymbalyuk, G.S., Gaudry, Q., Masino, M.A., Calabrese, R.L.: Bursting in leech heart interneurons: Cellautonomous and network-based mechanisms. J. Neurosci. 22(24), 10580-10592 (2002)

20. Bhalla, U.S., Bower, J.M.: Exploring parameter space in detailed single neuron models: simulations of the mitral and granule cells of the olfactory bulb. J. Neurophysiol. 69(6), 1948-1965 (1993)

21. Gunay, C., Edgerton, J.R., Jaeger, D.: Channel density distributions explain spiking variability in the globus pallidus: a combined physiology and computer simulation database approach. J. Neurosci. 28, 7476-7491 (2008)

22. Wenning, A., Cymbalyuk, G.S., Calabrese, R.: Heartbeat control in leeches. I. Constriction pattern and neural modulation of blood pressure in intact animals. J. Neurophysiol. 91, 382-396 (2004)

23. Calabrese, R.L.: The neural control of alternate heartbeat coordination states in the leech. J. Comp. Physiol. 122, 111-143 (1977)

24. Hodgkin, A.L., Huxley, A.F.: A quantitative description of membrane current and its application to conduction and excitation in nerve. J. Physiol. 117, 500-544 (1952)

25. Bower, J.M., Beeman, D.: The Book of GENESIS: Exploring Realistic Neural Models with the GEneral NEural SImulation System, 2nd edn., Springer (TELOS), New York (1998). http://www. genesis-sim.org/GENESIS/

26. Wikipedia: Brute-Force Search (2010). http://en.wikipedia.org/wiki/Brute-force_search

27. Wikipedia: Free Lossless Audio Codec (2010). Http://en.wikipedia.org/wiki/Free_Lossless_Audio_Codec

28. ModelDB (2010). http://senselab.med.yale.edu/ModelDB/

29. Marder, E., Goaillard, J.M.: Variability, compensation and homeostasis in neuron and network function. Nat. Rev. Neurosci. 7, 563-574 (2006) 